\title{
A new biarticular joint mechanism to extend stiffness ranges
}

\author{
Hannes Höppner ${ }^{1}$, Wolfgang Wiedmeyer ${ }^{1}$ and Patrick van der Smagt ${ }^{2}$
}

\begin{abstract}
We introduce a six-actuator robotic joint mechanism with biarticular coupling inspired by the human limb which neither requires pneumatic artificial muscles nor tendon coupling. The actuator can independently change monoarticular and biarticular stiffness as well as both joint positions. We model and analyse the actuator with respect to stiffness variability in comparison with an actuator without biarticular coupling. We demonstrate that the biarticular coupling considerably extends the range of stiffness with an 70-fold improvement in versatility, in particular with respect to the end-point Cartesian stiffness shape and orientation. We suggest using Cartesian stiffness isotropy as an optimisation criterion for future underactuated versions.
\end{abstract}

\section{INTRODUCTION}

Variable Stiffness Actuators (VSAs) are actively being researched because of their ability to absorb energy during highly dynamic impacts and their possibility to temporarily store elastic energy [1], [2], [3], [4], [5], [6], [7]. Furthermore, humans modulate impedance in order to meet accuracy demands during goal-directed arm movements [8]. However, all existing tendon-free VSAs are designed such that they are only able to change stiffness and position of one joint (monoarticular (mon.art.) actuators). In biology, however, one finds many multi-articulated skeletal muscles, crossing more than one joint and thus changing both stiffness and position of each joint when activated. A special case of these groups of muscles are biarticular (biart.) muscles, with the prominent examples biceps and triceps brachii, coupling shoulder and elbow. The role of these muscles is as follows:

- morphing endpoint stiffness-In [9, pp. 361-366] English et al. analysed the influence of biart. muscles based on a musculo-skeletal model of the human arm. They revealed that the loss of biart. muscles leads to a more elongated stiffness ellipse (strong preferred displacement direction), a reduced maximum stiffness, a reduced possible orientation range and finally the result that stiffness produced by double-joint muscles cannot be replaced by using only single-joint stiffness.

- stabilising the limb-McIntyre et al. discussed in [10] the role of destabilising forces at the endpoint

\footnotetext{
${ }^{1}$ These authors are with the Institute of Robotics and Mechatronics, German Aerospace Center (DLR), D-82234 Oberpfaffenhofen, Germany \{hannes.hoeppner, wolfgang.wiedmeyer\}@dlr.de

${ }^{2}$ Patrick van der Smagt is with the Department for Informatics, Technische Universität München, Germany
}

and their overall influence on joint torques. The amount of joint torque produced, e.g., in the elbow can affect the required stiffness at the shoulder. "... With multijoint muscles present, each muscle stiffness need be a function only of its own force output in order to maintain overall limb stability. The multiarticular muscles provide the necessary coupling between joints. This is an example of a mechanical design simplifying the control problem." The self-stabilising characteristics of biart. coupling were also shown by Iida et al. [11], for both running and walking gaits.

- independent control of force and positionKumamoto et al. showed [12] that biart. muscles allow for an independent control of endpoint force and position. Furthermore, they found that these muscles allow for an independent control of endpoint stiffness and output force direction.

- open-loop control of position-In [13] Taub et al. showed that monkeys with deafferent limbs were able to reach visual targets with and without visual feedback of limbs position. Consequently, $\mathrm{Ku}-$ mamoto et al. concluded [14] that biart. muscles allow for precise, smooth and rapid movement patterns of the endpoint without the use of any positional feedback. They concluded that "...the arbitrary control of the output force direction, and elastic and viscous ellipses ... can move the endpoint precisely to any desired target point without use of a positional feedback signal, that is a possibility of an open loop control."

- transferring energy-biart. muscles provide the capability to transport the mechanical output mainly produced by mon.art. muscles to joints where it can effectively contribute to the desired aim of movement [15]. E.g., during running, instead of braking the knee using mon.art. muscles and dissipating the energy in form of heat, the biart. muscles provide the possibility to optimally transfer the energy to the hip in order to maintain the forward motion.

In this paper we will present and analyse a two-joint planar VSA with and without biart. coupling based on the Bidirectional Antagonism with Variable Stiffness (BAVS). The actuator BAVS, which is neither tendondriven (possible drawback: tendon creeping) nor based on artificial pneumatic muscles (possible drawback: highly nonlinear), was introduced in [16]. The focus is to investigate the ability to adapt passive (intrinsic) endpoint 
stiffness at zero net force. We will compare and discuss these two different types of VSA systems in a simulation study in order to show what will be lost and what can be gained in a system with biart. coupling. Therefore we will set out to investigate the full ability to change passive stiffness and propose a method that allows us to do so.

\section{RELATED WORK}

The use of biarticularity in robotic design is nothing new. Different implementations have been proposed: either by tendon-driven actuation with base-fixed motors [11], [17], [18], [19], by pneumatic artificial muscles [12], [20], [21], by planetary gears [22], [23], [24], by pulleys [25], or by directly coupling a linear motor with the end effector [26]. However, even if some of these actuators are able to change their active (controlled) stiffness (e.g., impedance control [26] or stiffness control for disturbance rejection [23], [25]), not all of these actuators are designed to change their passive (intrinsic) stiffness [19], [22], [23], [24], [25], [26] even when actuated by six actuators for two joints [18].

One of the main objectives of many studies is to show what is gained with biart. coupling with respect to endpoint force production [18], [20], [24], [25], [27]. It was shown that with biart. coupling the shape of the maximum output force distribution at the endpoint becomes more homogeneous and changes from quadrangular to hexagonal.

A view also concentrated on the ability to change passive stiffness [12], [14], [17], [28]. In [17] Kadota et al. built a tendon-based robotic platform with biart. coupling called HIPRO. HIPRO consists of six actuators arranged in an antagonistic manner with nonlinear elastic elements for a planar robotic system with two DoF. It was shown that the system can vary the position of the endpoint with negligible effects on the orientation of the Cartesian endpoint stiffness ellipse ${ }^{1}$.

In [12] and [14], Kumamoto et al. investigated the capabilities of a robotic planar arm with two DoF actuated by six pneumatic artificial muscles. As mentioned before the authors showed that a biart. coupling will positively contribute to the compliant properties and allow for independent control of either endpoint position and force or endpoint force direction and stiffness. Furthermore it was shown that this coupling will lead to smooth, fine, and precise movement patterns of the endpoint.

Another study which concentrates on stiffness ellipse control using VSA mechanisms with biart. coupling was presented by Kashiwagi et al. [28]. They proposed a control method called hybrid stiffness ellipse control which merges mechanical and controlled stiffness of a tendonbased two-DoF planar robotic actuator with six musclelike VSA and ideal exponential elastic elements. Biart.

\footnotetext{
${ }^{1}$ Unfortunately, the paper is written in Japanese, and we were not able to fully understand it.
}

coupling was provided in order to be able to change all elements of the mechanical joint stiffness matrix. With simulated perturbations they showed that their control method is always able to adapt to a desired control stiffness, while the initial response is dominated by the adjusted intrinsic mechanical stiffness.

\section{TYPES}

\section{A. Principle of $B A V S$}

Both types of VSAs that we analyse in this paper have in common that they are based on the principle that we call Bidirectional Antagonism with Variable Stiffness (BAVS) [16], which is implemented in the forearm rotation and both wrist joints of the DLR Hand-Arm System [6]. Fig. 1 (top) shows a simplified version, in which the motors can be turned around their motor axis instead of being fixed to a base frame. The two motors are coupled with the base via nonlinear elastic elements. Both motors can both push and pull at the drive side of the joint and assist each other, for which reason we use the term helping antagonism. The position of the joint

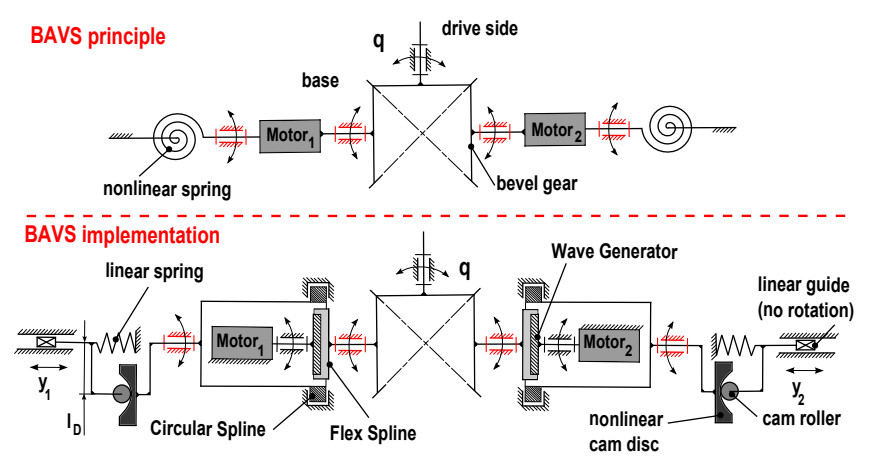

Fig. 1. Principle of Bidirectional Antagonism with Variable Stiffness - The upper figure shows a simplified representation of BAVS, the lower figure the version of a double spring solution with symmetric cam discs. Note that for reasons of presentability the two cam discs are depicted orthogonal to the image plane.

can be changed by identical motor movement, while its stiffness is changed by an opposed motor movement. Its realisation as it is implemented in the DLR Hand-Arm System is shown in Fig. 1 (bottom). The two nonlinear elastic elements are realised using a combination of a linear spring and a cam disc with a nonlinear relation between its rotation and the resulting spring deflection. In order to fix the motors to the base frame and to use their full power, a Harmonic Drive is used with a bedded circular spline, which is coupled to the nonlinear elastic element (arrangement of the harmonic drive similar to a planetary gear).

In [16] we also introduced different variations of BAVS as they are a double/single spring solution and asymmetric/symmetric cam disc shape. We showed that asymmetric cam disc shape and a double spring solution as it is implemented in the forearm-rotation of the DLR Hand-Arm System (see Fig. 2) has several advantages in 
comparison to single spring solution with symmetric cam disc shape.

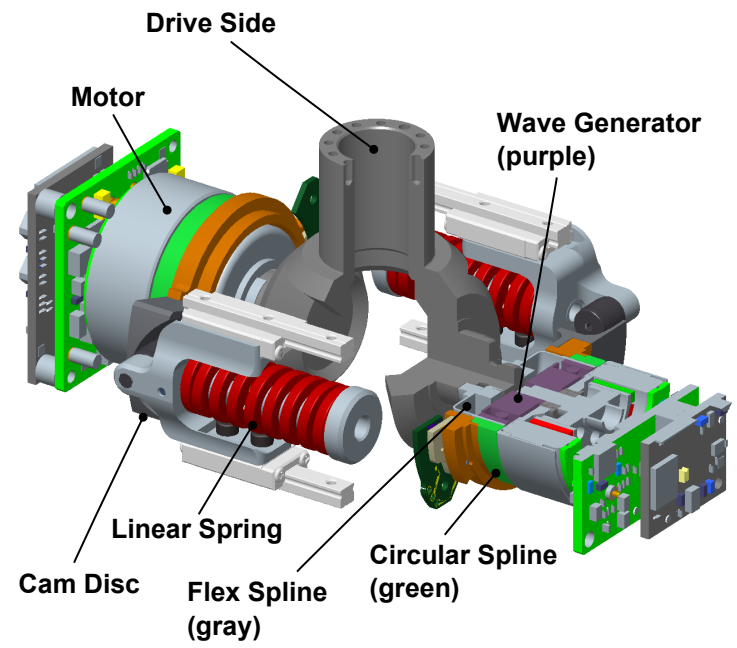

Fig. 2. Bidirectional Antagonism with Variable Stiffness as it is implemented in the forearm rotation of the DLR Hand-Arm System with asymmetric cam disc shape and double spring solution.

The joint stiffness $r$ can be calculated by

$$
r=\frac{\partial \tau}{\partial q},
$$

where $\tau$ and $q$ are joint torque and its externally incurred deflection. The torque depends on the spring stiffness $c_{F}=22.1 \mathrm{~N} / \mathrm{mm}$, the moment arm of the cam disc $l_{D}=21.65 \mathrm{~mm}$ and the deflection of the springs caused by the cam disc shape $y_{1 / 2}$ and its slope $y_{1 / 2}^{\prime}$ :

$$
\begin{gathered}
\tau(\sigma, q)=c_{F} l_{D}\left(y_{1} y_{1}^{\prime}+y_{2} y_{2}^{\prime}\right) \\
y_{1}=f(\sigma+q) \quad y_{2}=f(\sigma-q) .
\end{gathered}
$$

The symmetric cam disc shape is modelled by

$$
y_{1 / 2}(\sigma \pm q)=R-\sqrt{R^{2}-\left(r_{D}(\sigma \pm q)\right)^{2}}+y_{0},
$$

where $R=8.2 \mathrm{~mm}$ is the radius of the symmetric cam disc, $\sigma$ the pretension of the joint in ${ }^{\circ}$ and $y_{0}=2 \mathrm{~mm}$ the initial pretension of the spring. The maximum deflection of each cam disc is limited by $\pm 18^{\circ}$ being the maximum deflection of the joint around an equilibrium position.

\section{B. mon.art. VSA system}

The mon.art. VSA system that we analyse here is a planar robotic system with two DoF, elbow and shoulder joint, which joints are based on the BAVS principle explained above. The system has no biart. coupling. Unlike the implementation in the DLR Hand-Arm System (see Fig. 2), a double-spring solution with symmetric cam disc shape is chosen for both joints, thus simplifying the comparability for the planned analysis. In this joint configuration the maximum stall torque of a single joint is $\pm 8 \mathrm{Nm}$ and the maximum joint stiffness $127.6 \mathrm{Nm} / \mathrm{rad}$.

\section{C. biart. VSA system}

The biart. VSA system is in terms of the mon.art. joints identical to the mon.art. system and basically consists of two BAVS joints with a double-spring solution and symmetric cam discs. New is its biart. coupling (see red dashed regions in Fig. 3). The two joints are coupled using two series of nonlinear elastic elements and rotatory motors. The biart. stiffness is changed by opposed movement of the biart. coupling motors 3 and 4 . In contrast to, e.g., the human legs, this configuration is able to adjust both joint positions and mon.art. as well as biart. joint stiffnesses independently. This is realised through the cam disc-roller combination - contrary to the mon.art. BAVS - by rotating about the motor axis without changing their relative orientations. Thus, by identical movements of motors 3 and 4 the joint positions can be changed without changing the biart. or mon.art. stiffnesses.

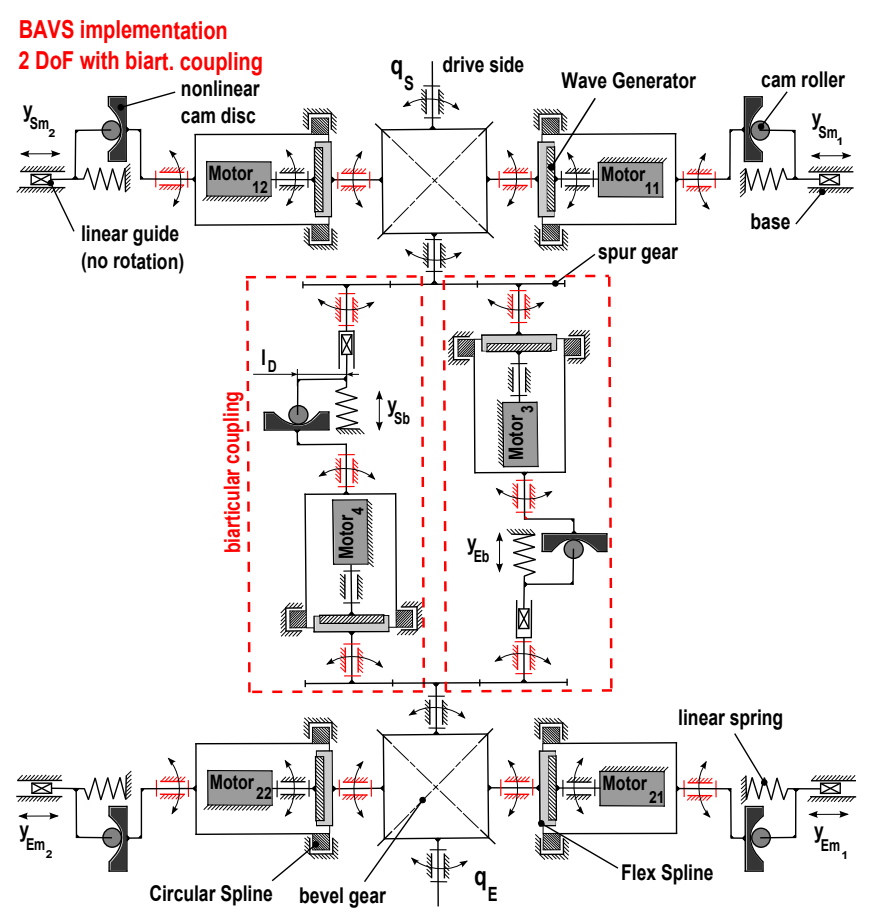

Fig. 3. A biarticular VSA system with six actuators and two DoF-The two drive sides of the arm are at the bottom and top of each figure. Imagine the depicted base as the upper arm of an elbow-shoulder configuration.

In order to compare the mon.art. and biart. VSA systems in terms of stiffness adaptability equally, identical components (same motors, springs and cam discs) as for the mon.art. joints are used: the motor is fixed to the frame using a Harmonic Drive with a bedded circular spline; the nonlinear elastic element is again realised by a combination of symmetric cam discs and linear springs. The symmetric cam disc shape used is indeed not the optimal configuration presented in [16]. But if an asymmetric cam disc shape would be used, different behaviour depending on ratios of pretension of biart. 
and mon.art. actuators could emerge. Thus it would be difficult to keep the joint position constant during cocontraction of the biart. elastic elements.

\section{AnAlysis}

In this section we will model the two systems and compare them with respect to adjustable static stiffness; no effects of damping and inertia are considered. Even if the difference between the two mechanisms would be more obvious in joint space because the biart. VSA is also able to adjust off-diagonal joint stiffness terms, we will compare them in Cartesian space. We think that this way of comparison is more intuitive due to its comparability to human measurements, e.g. [29]. The analysis of the different types is done by modelling a planar two-DoF arm systems with shoulder $(\mathrm{S})$ and elbow (E) joints (see Fig. 4).

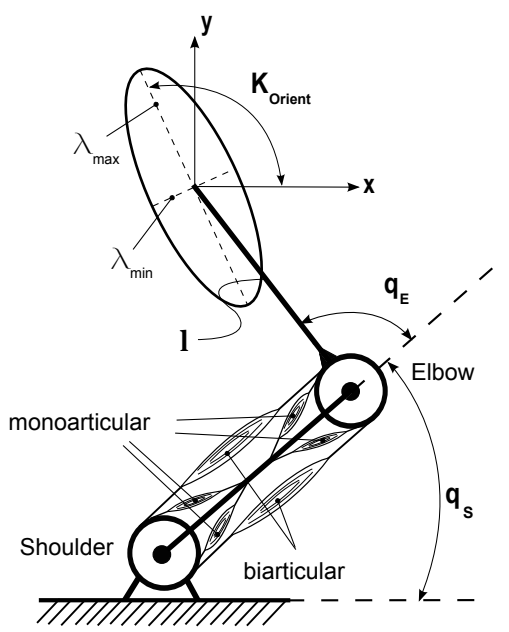

Fig. 4. Simplified planar arm model consisting of the two joints shoulder and elbow driven by six actuators, four monoarticular and two biarticular muscles. The two compared systems are either equipped or not with a biart. VSA coupling. Furthermore, the orientation $K_{\text {orient }}$ of the considered Cartesian stiffness ellipse and its eigenvalues $\lambda_{\min }$ and $\lambda_{\max }$ are depicted.

\section{A. Model}

The torque at the shoulder $\tau_{S}$ and elbow $\tau_{E}$ joint can be calculated by

$$
\tau_{S / E}=\tau_{S / E_{\text {mon }}}+\tau_{\text {biart }}
$$

where $\tau_{S / E_{\text {mon }}}$ is the torque produced by the mon.art. and $\tau_{\text {biart }}$ the torque produced by the biart. actuators at the shoulder and elbow joint. As we discussed in Section III the torque produced by a BAVS with a double spring solution can be calculated by

$$
\begin{gathered}
\tau_{S_{\mathrm{mon}}}\left(\sigma_{m_{S}}, q_{S}\right)=c_{F} l_{D}\left(y_{S_{m 1}} y_{S_{m 1}}^{\prime}+y_{S_{m 2}} y_{S_{m 2}}^{\prime}\right) \\
\tau_{E_{\mathrm{mon}}}\left(\sigma_{m_{E}}, q_{E}\right)=c_{F} l_{D}\left(y_{E_{m 1}} y_{E_{m 1}}^{\prime}+y_{E_{m 2}} y_{E_{m 2}}^{\prime}\right),
\end{gathered}
$$

where $\sigma_{m_{S / E}}$ are the pretension and $q_{S / E}$ the external deflections of the shoulder and elbow joint. $y_{m}$ and $y_{m}^{\prime}$ are the deflections of the springs and the correspondent cam disc slope (see Fig. 3). The torque of the biart. actuators can be derived by

$$
\tau_{\text {biart }}\left(\sigma_{b}, q_{S}, q_{E}\right)=c_{F} l_{D}\left(y_{S_{b}} y_{S_{b}}^{\prime}+y_{E_{b}} y_{E_{b}}^{\prime}\right),
$$

and depends on the biart. pretension $\sigma_{b}$ and the external joint deflections $q_{S / E}$.

In general, the joint stiffness matrix can be calculated by $R=\partial T / \partial Q \in \mathbb{R}^{n \times n}$, where $T$ and $Q$ are the joint torques and the external joint deflections; $n$ the number of joints. Because the stiffness of each joint can be changed, $R$ is a square matrix. For the systems considered here this matrix equals

$$
\begin{aligned}
R & =\left[\begin{array}{ll}
r_{S S} & r_{S E} \\
r_{E S} & r_{E E}
\end{array}\right] \\
& =\left[\begin{array}{cc}
r_{S_{\text {mon }}}+r_{\text {biart }} & r_{\text {biart }} \\
r_{\text {biart }} & r_{E_{\text {mon }}}+r_{\text {biart }}
\end{array}\right] .
\end{aligned}
$$

The first index of the scalar entries of (7) indicates the entry of $T$, the second the entry of $Q$ for the partial differentiation (cf. [29]). Note that for the mon.art. VSA no joint coupling is provided and thus the joint stiffness has no off-diagonal terms $r_{S E}=r_{E S}=0$. If stiffness is computed around an equilibrium position, a mapping between joint and Cartesian stiffness can be achieved by

$$
R=J(Q)^{T} K J(Q),
$$

where $J(Q)$ is the Jacobian, which is a $2 \times 2$ matrix for the hand-arm model [30]. Both stiffness matrices $R$ and $K$ are symmetric because the two systems are modelled as conservative systems. Eq. (8) can be rewritten as

$$
K=J(Q)^{-T} R J(Q)^{-1} .
$$

Because $J(Q)$ is square for the considered model its inverse can be computed. The Jacobian is obtained by

$$
\begin{array}{r}
J(Q)=\left[\begin{array}{cc}
-l_{S} s_{S}-l_{E} s_{S E} & -l_{E} s_{S E} \\
l_{S} c_{S}+l_{E} c_{S E} & l_{E} c_{S E}
\end{array}\right] \\
s_{S}=\sin q_{S} \quad s_{S E}=\sin \left(q_{S}+q_{E}\right) \\
c_{S}=\cos q_{S} \quad c_{S E}=\cos \left(q_{S}+q_{E}\right),
\end{array}
$$

and contains the information about the geometrical relations between the joints. $q_{S}$ and $q_{E}$ are the angles describing the position of the shoulder and elbow, $l_{S}$ and $l_{E}$ are the link length of the upper and lower arm, respectively.

\section{B. Comparison of the eigenvalues in Cartesian space}

It is obvious that the biart. actuator can reach larger stiffness ranges than the mon.art. VSA, because the biart. coupling increases off-diagonal as well as diagonal terms of the joint stiffness matrix $R$ (see Eq. (4)-(7)). In order to show what can be gained with respect to the biart. coupling rather than showing how more actuators will increase the stiffness we limited the compared Cartesian stiffness space to the maximum/ minimum range that the mon.art. actuator is able to reach $\left(4 \leq K_{x x} \leq\right.$ $99 \mathrm{~N} / \mathrm{m},-99 \leq K_{x y / y x} \leq-4 \mathrm{~N} / \mathrm{m}$ and $20 \leq K_{y y} \leq$ 


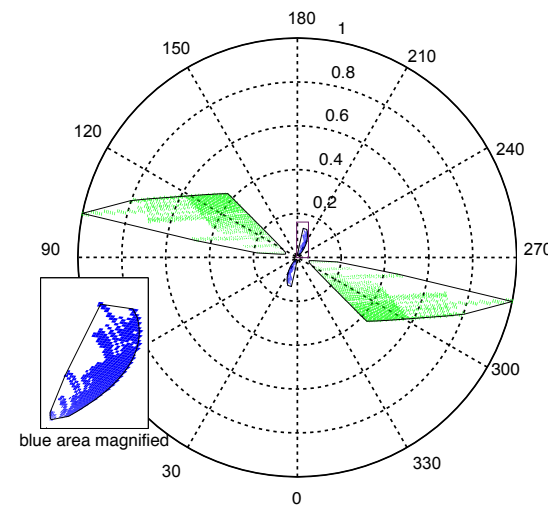

(a) mon.art. VSA

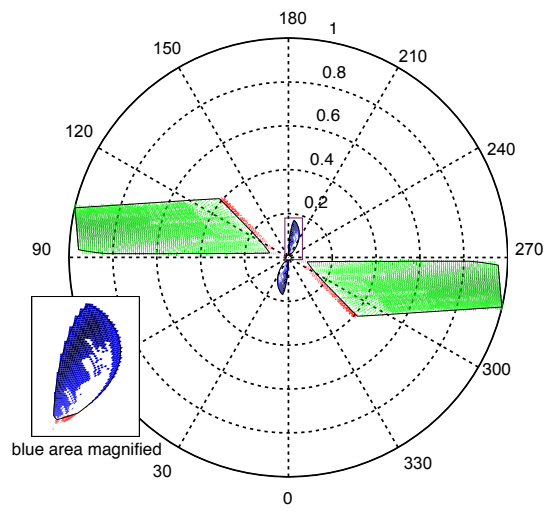

(b) difference between mon.art. \& biart.

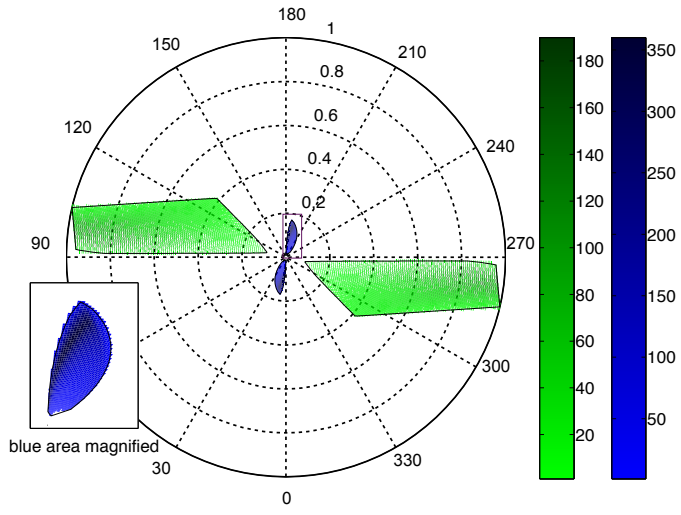

(c) biart. VSA

Fig. 5. Comparison of the Cartesian stiffness $K$ that can be reached by the two systems and their difference- The eigenvalues of the (symmetric) Cartesian stiffness matrix and the orientation of the corresponding eigenvector are depicted. In Fig. 5(a) and 5(c) the larger eigenvalue $\lambda_{\max }$ is depicted in green, the small eigenvalue $\lambda_{\max }$ in blue in form of a distance to the centre of the polar plot. The radius of the polar plot is normalized to range between 0 and 1 where a radius of 1 corresponds to the biggest $\lambda_{\max }$. The polar angle of each eigenvalue corresponds to the respective orientation. The darker a colour of a segment the more often this eigenvalue was reached. The black line in each plot corresponds to the convex hull. The comparison in Fig. 5(b) shows in green and blue, which points can only be reached by the biart. system, and in red which only the mon.art. system can reach. The mon.art. VSA can reach $0.2 \%$ of the $10^{6}$ Cartesian stiffness combinations, whereas the biart. VSA reaches $14.5 \%$. The area of the convex hull enclosing the larger eigenvalue of the biart. system is 1.6 times as large; the area of the convex hull enclosing the smaller eigenvalue twice as large as the mon.art. system.

$498 \mathrm{~N} / \mathrm{m})$. Thus we varied the Cartesian stiffness matrix $K$ at the end effector within the achievable range of Cartesian stiffness the mon.art. actuator is able to reach instead of varying stiffness in joint space. In order to keep the analysis simple, we make following restrictions:

- The link lengths $l_{S}=l_{E}=l=0.8 \mathrm{~m}$ are identical.

- As it can be seen from Eq. (9) and (10), Cartesian stiffness $K$ can be adjusted by changing the joint position and keeping joint stiffness $R$ constant. Because the suggested VSA systems are able to adjust their joint stiffness independent from joint position we will compare them in a central position with a shoulder angle fixed at $45^{\circ}$ and an elbow angle of $90^{\circ}$.

- We will only allow changes in stiffness caused by cocontraction which can be preset by the VSA around an equilibrium position without any interaction with the environment. Changes in stiffness that will lead to a change in joint position or emerge from an external deflection of the joints will not be considered.

- Only co-contraction of respective antagonistic pairs will be allowed for the biart. VSA. Thus, it will not be possible to contract a biart. actuator against a mon.art. one and vice versa.

Co-contraction can be achieved by pretensioning the two mon.art. actuators $\sigma_{m_{S}}$ and $\sigma_{m_{E}}$ and for the biart. VSA additionally by pretensioning the biart. coupling $\sigma_{b}$. Because within this work we assume only stiffness around an equilibrium position without any external deflection, Eqs. (3), (5) and (6) only depend on the mentioned pretensions.

The (symmetric) Cartesian stiffness matrix $K$ was computed for $10^{6}$ different configurations. These Carte- sian stiffnesses were transformed to joint space and it was checked whether a corresponding set of combinations of mon.art. and biart. cam disc pretensions within the maximum deflection of the cam discs could be found. The transformation of the set into joint space will typically lead to non-zero off-diagonal joint stiffness terms. Since the mon.art. VSA can only vary diagonal joint stiffness terms of $R$ and a computation of $R$ out of varying $K$ will almost never lead to zero off-diagonal terms, we limited them to a corresponding maximum pretension of $0.1^{\circ}$ for the mon.art. VSA. This corresponds approximately to the accuracy within which the cam discs can be adjusted. If we would allow only exactly zero off-diagonal terms for the mon.art. system, the capability of this system would be disproportionately high underestimated. Note that, in a real system as well, e.g. due to static friction, the computation of $R$ out of a measured $K$ of a system without biart. coupling would most likely never lead to zero off-diagonal terms of $R$.

Following the representation in [30], we depict the distributions of Cartesian stiffnesses that can be reached by the two systems in Fig. 5. The mon.art. system is able to reach $0.2 \%$, the biart. VSA $14.5 \%$ of the $10^{6}$ Cartesian stiffness combinations. Fig. 5(b) shows the difference between the compared systems. It shows that the biart. coupling allows the system to reach many more eigenvalues of $K$ than just the mon.art. system is capable of $^{2}$. However, there are also sections which can only be reached by the mon.art. VSA. We will examine this more

\footnotetext{
${ }^{2}$ Note that, if we will use the same representation to depict the stiffness $R$ in joint space, the mon.art. system will only be able to reach values along the horizontal and vertical axis, while the biart. system will as well reach in-between stiffness combinations.
} 
closely below.

\section{Comparison of shape and orientation of endpoint stiffness}

Hogan introduced in [29] a singular value decomposition of the endpoint stiffness matrix $K$ to visualise the stiffness as an ellipse with its attributes size, shape and orientation as a way of graphical representation of the stiffness configuration at the endpoint. The characteristics shape and orientation can be considered as criteria of quality while the size is a criterion of quantity. Since it is quite obvious that the biart. VSA can generate higher stiffnesses with two more built-in springs, the focus in this paper is more on the quality rather than the quantity of stiffness that can be obtained by biart. springs.

The shape of the stiffness ellipse is computed by

$$
K_{\text {shape }}=\left|\frac{\lambda_{\max }}{\lambda_{\min }}\right|,
$$

and is a characterisation of isotropy of the endpoint stiffness. $\lambda_{\max / \min }$ are the eigenvalues and denotes the stiffness in the direction of the major and minor axis of the stiffness ellipse. $K_{\text {shape }}$ of 1 represents an ideal isotropic endpoint stiffness, i.e., the endpoint stiffness can be represented by a circle. It means that a force perturbing the endpoint in any direction would lead to an unique and proportional restoring force and displacement in exactly the opposite direction. Thus, it is reasonable to conclude that a $K_{\text {shape }}$ of 1 is a desirable property and can be used as a metric for stability, e.g., for holding a position in unstable force fields or in a situation of an expected load with unknown impact direction [31]. As Hogan discussed in [29] a mechanism without biart. coupling will never be able to achieve ideal isotropy at the endpoint, and with biart. coupling only in a limited space of an elbow angle larger than $90^{\circ}$ and less than $180^{\circ}$. Using equations (8) and (10) and the condition for isotropy at the endpoint [29]

$$
K=\left[\begin{array}{cc}
-k & 0 \\
0 & -k
\end{array}\right],
$$

it follows that the mechanism must hold the following condition in joint space:

$$
R=\left[\begin{array}{cc}
r_{S S} & r_{S S} / 2 \\
r_{S S} / 2 & r_{S S} /\left(2+2 \cos \left(q_{E}\right)\right)
\end{array}\right],
$$

to achieve isotropy at the end effector. With (7) it follows that $r_{\text {biart }}=2 r_{S_{\text {mon }}}$. The condition shows that the joint stiffness of the biart. coupling must be twice the stiffness of the mon.art. shoulder actuator to achieve isotropy at the endpoint. This condition is somewhat similar to the fact that the biart. muscles in the human arm are much stronger compared to the mon.art. muscles. On the other hand, a large $K_{\text {shape }}$ can be desirable in reaching movements with a perpendicular instable force field [32], where high stiffness is required in the direction of the unstable force field and low stiffness in the direction of

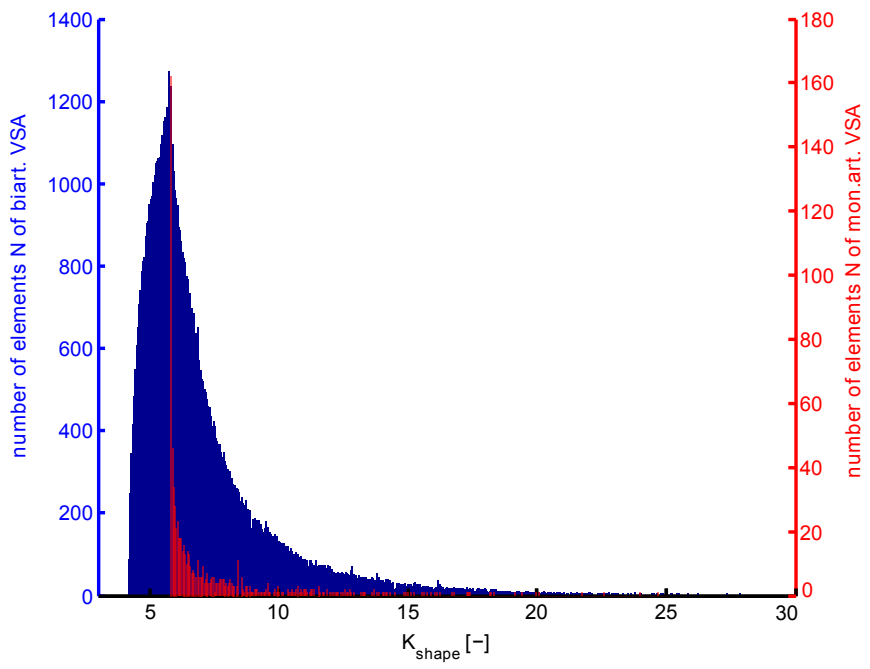

Fig. 6. Histogram of occurrences of $K_{\text {shape }}$ for the reached $K$ of the $10^{6}$ Cartesian stiffness combinations.

the movement in order to stay on the track. All in all, it can be concluded that a wide range for $K_{\text {shape }}$ with a minimum close to 1 is desirable.

In Fig. 6 the distribution of $K_{\text {shape }}$ of the reachable Cartesian stiffness combinations for both actuator types is depicted in form of a histogram showing how often which $K_{\text {shape }}$ can be reached by either mechanism. It shows that the minimum $K_{\text {shape }}$ that can be reached by the mechanism with biart. coupling is 4.2 and without 5.8. Additionally, the mechanism with biart. coupling can reach values up to 29.0 while the mon.art. system has an upper limit of 25.1. The minimum $K_{\text {shape of the }}$ mon.art. VSA is also its most often occurring $K_{\text {shape }}$ and one of the most occurring values of the biart. VSA and belong to Cartesian stiffness combinations, where a large mon.art. shoulder joint stiffness $r_{S S}$ and a small elbow joint stiffness $r_{E E}$ is required. However, even if biart. coupling is provided a $K_{\text {shape }}$ of 1 cannot be reached. Further analysis showed that the minimum $K_{\text {shape }}$ of the biart. VSA remains the same even if the Cartesian space is not limited to the mon.art. stiffness. Nevertheless, in the proposed biart. mechanism we want to be able to use the same springs for both, for the mon.art. actuators as well as for the biart., leading to a minimum $K_{\text {shape }}$ unequal to 1 .

The orientation of the stiffness ellipses can be calculated using the definition of the dot product in Euclidean space

$$
K_{\text {orient }}=\arccos \left(\left(\begin{array}{l}
1 \\
0
\end{array}\right) \cdot v\right),
$$

where $v$ denotes the normalized eigenvector corresponding to $\lambda_{\max }$ and $K_{\text {orient }}$ is the angle between the positive $x$-axis and $v$ (see Fig. 4). Fig. 7 shows the distribution of $K_{\text {orient }}$ that could be reached by the two mechanisms (similar to Fig. 6). It shows that the biart. system covers a range of about $39.2^{\circ}$ and the mon.art. system of about $36.2^{\circ}$ of different $K_{\text {orient }}$. However, in contrast 


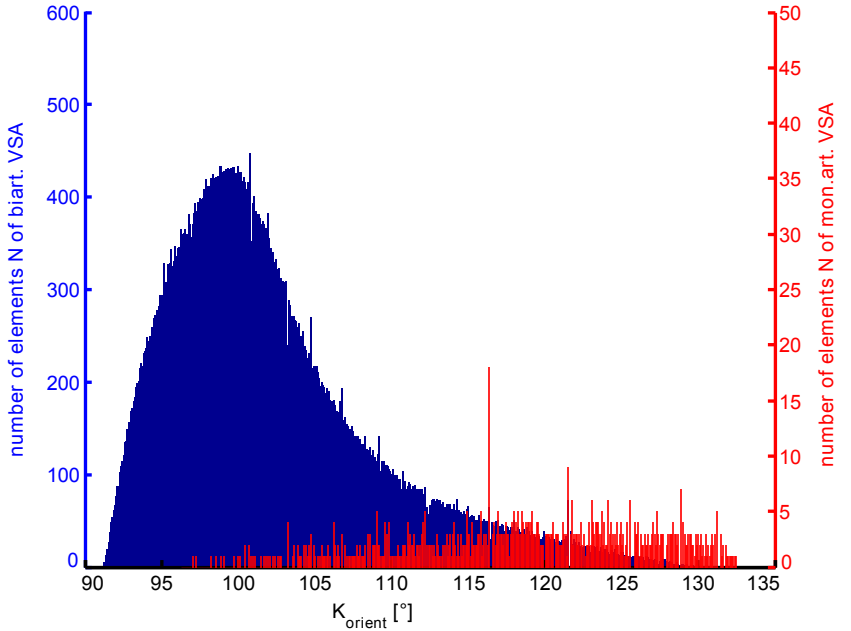

Fig. 7. Histogram of occurrence of $K_{\text {orient }}$ for the reached $K$ of the $10^{6}$ Cartesian stiffness combinations.

to $K_{\text {shape }}$, there are a few orientations up to $2.1^{\circ}$ higher than the largest orientations of the biart. VSA which appear either generally or more frequently be used by the mon.art. actuator. So why does the biart. VSA, which has the same mon.art. properties, but additionally biart. coupling, not reach these orientations? This is caused by the pretension $y_{0}$ of the biart. springs, similar to the mon.art. springs. This pretension causes a positive shift in the stiffness $r_{S S}$ and $r_{E E}$ in a way that the difference between $r_{S S}$ and $r_{E E}$ decreases. As a result the orientation of the stiffness ellipse changes. Thus, if it is favoured to reach also the higher orientations, the pretension of the biart. springs had to be reduced, but it has to be considered that these larger orientations usually belong to a high $K_{\text {shape }}$ (extremely elongated stiffness ellipse).

\section{Discussion and Conclusion}

In this work we proposed a mechanism of a VSA with biart. coupling. As the biart. mechanism is based on BAVS principle, which we introduced in [16], the system is able to independently change endpoint stiffness and position. Furthermore, a change in mon.art. stiffness will not lead to a change in biart. stiffness and vice versa, thus giving more control stability.

In our simulation study we investigated how a system with vs. without biart. coupling can reach a range of Cartesian endpoint stiffnesses. To this end, we used a method to plot a multiple set of stiffness configurations described in [30] to compare different planar VSA mechanisms. With respect to stiffness reachability, we found that biart. coupling considerably extends the system capabilities by a 70-fold improvement in versatility and can therefore be strongly favoured over mon.art. ones.

The range of orientations and shapes of Cartesian endpoint stiffness ellipses that the proposed biart. actuator can reach is slightly larger than that of the purely mon.art. system. Additionally, with $39.2^{\circ}$ it is larger than found in humans studies at $30^{\circ}$ [33], [34], [35]. Interestingly, we found that the mon.art. mechanism reaches stiffness combinations that the biart. VSA cannot reach; this can be explained by the pretension of the biart. springs.

It has to be acknowledged that more complex principles can enrich biart. manipulator behaviour. For instance, allowing to preload the biart. actuator against a mon.art. one, leading to non-symmetric co-contraction. Furthermore, if the system has been designed such that the strength of the biart. springs is twice the strength of the mon.art. ones, endpoint stiffness isotropy can be achieved. This can be helpful to absorb the energy of an expected load with unknown impact direction. However, in order to compare both actuator types equally and to keep the extensive calculation simple, we refrained from these possibilities.

As already stated in the introduction, the design neither requires the use of pneumatic artificial muscles nor tendon-coupling, which can be an advantage if one aims to avoid non-linearities due to tendon creeping, compressibility of air, and hysteresis effects of viscoelastic materials. Conversely, we cannot claim that our approach is universally optimal; for instance, in comparison to tendon driven systems it increases inertia at the drive side of the joint.

Suitable applications for this actuator are primarily humanoid robotics, in particular in constructing versatile arms and legs, but the approach is also useful for industrial robotics. As we initially stated, the biart. coupling will stabilise the locomotion system obtaining inherent mechanical stability, thus simplifying the control problem. Furthermore, the possibility to transfer energy between joints can be used to reduce the amount of required energy during highly dynamic tasks; the increased stiffness range allows the system assume bandwidth-dependent optimal stiffness. This is useful for, e.g., switching between running and walking gaits or in a pick-and-place task. On the other hand, the wide range of stiffnesses allows the system to be highly precise in a static positioning tasks, by being stiff in one direction and flexible in another.

Finally, it has to be acknowledged that the biart. coupling leads to mechanical complexity. The additional gears and motors introduce more backlash, tolerances, and friction issues. Implementing 6 motors for a planar 2-DoF movement seems like a mechanical overkill. Thus, in future work we want to address different underactuated versions of the proposed actuator and optimise the stiffness ratios in mon.art. as well as for the biart. actuators, e.g., with respect to ranges of Cartesian endpoint stiffness ellipse orientations and shapes and with respect to its isotropy. 


\section{REFERENCES}

[1] T. Morita and S. Sugano. Design and development of a new robot joint using a mechanical impedance adjuster. In IEEE Int. Conf. on Robotics and Autom., pages 2469-2475, 1995.

[2] C. E. English and D. Russell. Implementation of variable joint stiffness through antagonistic actuation using rolamite springs. Mechanism and Machine Theory, 34(1):27-40, 1999.

[3] A. Bicchi and G. Tonietti. Fast and soft arm tactics: dealing with the safety-performance trade-off in robot arms design and control. IEEE Robotics and Automation Mag., 11:22-33, 2004.

[4] S. Migliore, E. Brown, and S. Deweerth. Biologically inspired joint stiffness control. In Proc. IEEE International Conference on Robotics and Automation, pages 4519-4524, 2005.

[5] Markus Grebenstein and Patrick van der Smagt. Antagonism for a highly anthropomorphic hand-arm system. Advanced Robotics, 22(1):39-55, January 2008.

[6] M. Grebenstein, A. Albu-Schaffer, T. Bahls, M. Chalon, O. Eiberger, W. Friedl, R. Gruber, S. Haddadin, U. Hagn, R. Haslinger, H. Höppner, S. Jorg, M. Nickl, A. Nothhelfer, F. Petit, J. Reill, N. Seitz, T. Wimbock, S. Wolf, T. Wusthoff, and G. Hirzinger. The DLR hand arm system. In IEEE International Conference on Robotics and Automation (ICRA), pages 3175-3182, may 2011.

[7] David J. Braun, Florian Petit, Felix Huber, Sami Haddadin, Patrick van der Smagt, Alin Albu-Schaffer, and Sethu Vijayakumar. Robots driven by compliant actuators: Optimal control under actuation constraints. IEEE Transactions on Robotics, 99(5):1-17, 2013.

[8] Luc P.J. Selen, Peter J. Beek, and Jaap H. Dieën. Impedance is modulated to meet accuracy demands during goal-directed arm movements. Experimental Brain Research, 172(1):129$138,2006$.

[9] Chad English. Stiffness behaviour in two degree of freedom mechanisms. Ph.d. thesis, Carleton University, Ottawa, Ontario, dec 1999.

[10] J. McIntyre, F.A. Mussa-Ivaldi, and E. Bizzi. The control of stable postures in the multijoint arm. Experimental Brain Research, 110:248-264, July 1996.

[11] Fumiya Iida, Juergen Rummel, and Andre Seyfarth. Bipedal walking and running with spring-like biarticular muscles. Journal of Biomechanics, 41(3):656-667, 2008.

[12] M. Kumamoto, T. Oshima, and T. Yamamoto. Control properties induced by the existence of antagonistic pairs of biarticular muscles-mechanical engineering model analyses. Human Movement Science, 13(5):611-634, 1994.

[13] Edward Taub, Israel A. Goldberg, and Phyllis Taub. Deafferentation in monkeys: Pointing at a target without visual feedback. Experimental Neurology, 46(1):178-186, 1975.

[14] M. Kumamoto, T. Oshima, and T. Fujikawa. Control properties of a two-joint link mechanism equipped with mono- and biarticular actuators. In IEEE Int. Workshop on Robot and Human Interactive Communication, pages 400-404, 2000.

[15] G.-J. van Ingen Schenau. From rotation to translation: Constraints on multi-joint movements and the unique action of biarticular muscles. Human Movement Science, 8(4):301-337, 1989.

[16] Werner Friedl, Hannes Höppner, Florian Petit, and Gerd Hirzinger. Wrist and forearm rotation of the DLR hand arm system: Mechanical design, shape analysis and experimental validation. In IEEE/RSJ International Conference on Intelligent Robots and Systems, pages 1836-1842, 2011.

[17] Z. Fukai K. Kadota, K. Suzuki and T. Oda. Study on the basic robot platform model "HIPRO" using biarticular muscles functions - evaluation of biarticular muscles functions by the robot arm using VEA-(in japanese). In The Japan Society for Precision Engineering, Committee of Biological Control System and Its Applied Technology, workshop, pages 11-16, 2004.

[18] V. Salvucci, Y. Kimura, Sehoon Oh, and Y. Hori. BiWi: Biarticularly actuated and wire driven robot arm. In Proc. IEEE Intl. Conf. Mechatronics, pages 827-832, april 2011.

[19] Jan Babič. Biarticular Actuation of Robotic Systems, chapter 13, pages 251-270. InTech, february 2012.
[20] R. Niiyama and Y. Kuniyoshi. Design principle based on maximum output force profile for a musculoskeletal robot. Industrial Robot: An Int. Journal, 37(3):250-255, 2010.

[21] K. Radkhah, T. Lens, and O. von Stryk. Detailed dynamics modeling of BioBiped's monoarticular and biarticular tendondriven actuation system. In IEEE/RSJ International Conference on Intelligent Robots and Systems (IROS), pages 42434250 , oct. 2012

[22] Y. Kimura, Sehoon Oh, and Y. Hori. Novel robot arm with bi-articular driving system using a planetary gear system and disturbance observer. In IEEE International Workshop on Advanced Motion Control, pages 296-301, march 2010.

[23] Sehoon Oh, Y. Kimura, and Y. Hori. Reaction force control of robot manipulator based on biarticular muscle viscoelasticity control. In IEEE/ASME International Conference on $A d$ vanced Intelligent Mechatronics, pages 1105-1110, july 2010.

[24] A. Umemura, Y. Saito, and T. Haneyoshi. The rigidity of the bi-articular robotic arm with a planetary gear. In IEEE International Workshop on Advanced Motion Control, pages 490-495, march 2010.

[25] K. Yoshida, N. Hata, S. Oh, and Y. Hori. Extended manipulability measure and application for robot arm equipped with bi-articular driving mechanism. In 35th Annual Conference of IEEE Industrial Electronics, pages 3083-3088, nov. 2009.

[26] Y. Nakata, A. Ide, Y. Nakamura, K. Hirata, and H. Ishiguro. Hopping of a monopedal robot with a biarticular muscle driven by electromagnetic linear actuators. In Proc. IEEE International Conference on Robotics and Automation, pages 3153-3160, may 2012.

[27] M. Kumamoto, T. Oshima, and T. Fujikawa. Biarticular muscle as a principle keyword for biomimetic motor link system. In Proc IEEE-EMB Conf. Microtechnologies in Medicine and Biology, pages 346-351, 2002.

[28] H. Kashiwagi, F. Okumura, S. Komada, and J. Hirai. Stiffness ellipse control of tendon mechanisms with nonlinear springs. In Robotics and Biomimetics (ROBIO), 2011 IEEE International Conference on, pages 1261-1266, dec. 2011.

[29] Neville Hogan. The mechanics of multi-joint posture and movement control. Biological Cybernetics, 331:315-331, 1985.

[30] A. Albu-Schaffer, M. Fischer, G. Schreiber, F. Schoeppe, and G. Hirzinger. Soft robotics: what Cartesian stiffness can obtain with passively compliant, uncoupled joints? In Proc. IEEE/RSJ International Conference on Intelligent Robots and Systems, volume 4, pages 3295-3301, 2004.

[31] Theodore E. Milner. Contribution of geometry and joint stiffness to mechanical stability of the human arm. Experimental Brain Research, 143(4):515-519, 2002.

[32] David W. Franklin, Gary Liaw, Theodore E. Milner, Rieko Osu, Etienne Burdet, and Mitsuo Kawato. Endpoint stiffness of the arm is directionally tuned to instability in the environment. The Journal of Neuroscience, 27(29):7705-7716, 2007.

[33] E.J. Perreault, R.F. Kirsch, and P.E. Crago. Voluntary control of static endpoint stiffness during force regulation tasks. Journal of neurophysiology, 87(6):2808-2816, 2002.

[34] H. Gomi and R. Osu. Task-dependent viscoelasticity of human multijoint arm and its spatial characteristics for interaction with environments. Journal of Neuroscience, 18(21):89658978, 1998.

[35] Mohammad Darainy, Nicole Malfait, Paul L. Gribble, Farzad Towhidkhah, and David Ostry. Learning to control arm stiffness under static conditions. Journal of Neurophysiology, 92:3344-3350, July 2004. 